\title{
Satisfação dos usuários do Portal de Periódicos da Capes: um estudo sobre a obtenção de sucesso no uso do sistema
}

Beatriz Valadares Cendón

\begin{abstract}
Professora associada da Escola de Ciência da Informação da UFMG
\end{abstract}

Juliana Lopes de Almeida Souza

Mestranda da Escola de Ciência da Informação da UFMG

Nádia Ameno Ribeiro

\section{Bolsista de Iniciação Científica do CNPq. Graduanda em Biblioteconomia da Escola de Ciência da Informação da UFMG}

A pesquisa, aqui apresentada, buscou verificar o grau de sucesso de usuários do Portal CAPES, na busca por um periódico específico e as razões para os casos de insucesso. A metodologia utilizada foi quantitativa, com coleta de alguns dados qualitativos. O estudo analisou, utilizando estatística descritiva, duas questões de um questionário sobre a satisfação dos usuários do Portal, as quais foram respondidas por 930 docentes de 17 universidades, das áreas de Ciências Biológicas, Ciências Humanas e Linguística, Letras e Artes. Os resultados mostram que 645 (70\%) dos pesquisadores, quase sempre ou frequentemente, obtêm sucesso ao procurarem por um periódico específico no Portal. Os pesquisadores menos bem sucedidos são os da área de Linguística, Letras e Artes. Para $76 \%$ dos pesquisadores, a razão principal para a não obtenção de sucesso é a indisponibilidade do periódico no Portal. Respostas às questões abertas mostraram, também, que, mesmo estando o periódico no Portal, especialmente para literatura mais antiga, pode ocorrer a falta do fascículo específico buscado ou, ainda, estando o fascículo presente no Portal, o acesso ao texto completo do artigo procurado pode não ser permitido. Razões para insucesso na busca relacionadas a fatores, tais como usabilidade, dificuldades com idiomas, falta de treinamento e dificuldades de acesso, foram menos citadas pelos respondentes. 
Palavras-chave: Estudos de uso e usuários; Satisfação do usuário de informação; Portal de Periódicos Capes; Biblioteca digital.

\section{Satisfaction of the user of the Capes Portal of Scientific Journals: a study on the success in the use of the system}

This paper evaluates the level of success of users of the CAPES Digital Library of Scientific Journals, when searching for a specific journal and the reasons for their failures. The methodology used was quantitative with some qualitative data collected. The study used descriptive statistics to analyze two questions of a survey concerning the Portal's user satisfaction which were answered by 930 faculty members of 17 universities in Brazil. The areas of knowledge investigated were Biological Sciences, Humanities and Linguistics, Literature and the Arts. The results show that 645 (70\%) of the researchers are almost always or frequently successful when searching for a specific journal in the Portal. Researchers in Linguistics, Literature and the Arts are the least successful. For $76 \%$ of the researchers, the main reason for not obtaining success is the absence of the journal in the collection. Responses to open questions reveal that, especially for older literature, the journal might be present in the collection but the specific issue needed may be absent or access to full text of the particular article sought may be restricted. Reasons for failure related to factors such as usability, difficulty with foreign languages and lack of training or difficulties for access were less cited by respondents.

Keywords: Use and user studies; user satisfaction; Capes Portal of Scientific Journals; Digital library.

Recebido em11.03.2011 aceito em 01.06.2011

\section{Introdução}

O Portal de Periódicos da CAPES é a maior biblioteca digital de periódicos científicos no Brasil, oferecendo, em fevereiro de 2011, acesso 
a 26.449 publicações periódicas internacionais e nacionais, além de renomadas bases de dados de resumos em todas as áreas do conhecimento. No contexto dos países de língua latina, o Portal de Periódicos é a experiência mais antiga, possuindo o maior número de instituições beneficiárias, bem como o conteúdo mais diversificado. Costuma ser tomado como um modelo pelos outros consórcios da região e é estratégico para o desenvolvimento de conhecimento no país.

Tendo em mente a relevância estratégica do Portal de Periódicos da CAPES, o corrente trabalho analisa a satisfação dos seus usuários, por meio do grau de sucesso obtido por docentes e pesquisadores na busca por periódicos no Portal, bem como as razões para os casos de insucesso. Os dados para este estudo foram levantados através de um questionário coletado via internet (web survey), enviado para 17 universidades federais e respondido por 930 docentes.

O artigo é iniciado com um relato sobre o surgimento e a evolução das bibliotecas digitais de periódicos eletrônicos, desenvolvimentos esses que possibilitaram a criação do Portal de Periódicos da CAPES. Segue-se uma discussão sobre os conceitos de sucesso de sistemas de informação e satisfação de seus usuários, construtos que buscam avaliar se uma biblioteca digital cumpre seu papel no atendimento das necessidades informacionais dos seus usuários. São apresentados, em seguida, a metodologia do estudo e os resultados da pesquisa.

\section{Evolução das bibliotecas digitais de periódicos eletrônicos}

Os periódicos científicos, cujo surgimento data da segunda metade do século XVII, são considerados um dos principais veículos de comunicação e divulgação do conhecimento científico (KING E TENOPIR, 1999; MEADOWS, 1999; MILLER, 2002; KLING; CALLAHAN, 2002). Recentemente, os estudos de Tenopir (2003) reafirmam essa constatação, mostrando que os artigos de periódicos são tidos, pelos cientistas americanos, como a fonte de informação mais importante para suas pesquisas.

Desenvolvimentos atuais permitiram a disponibilização do texto completo dos periódicos científicos em formato digital, os quais passaram, nesta forma, a serem denominados periódicos eletrônicos. Segundo Peek e Pomerantz (1998), o primeiro periódico científico a ser publicado em formato eletrônico foi o Chemical Titles, um serviço de alerta do Chemical Abstract Service (CAS), produzido em fita magnética, em 1962. Entretanto, os primeiros experimentos para criação de revistas científicas eletrônicas com padrões editoriais ocorreram em 1979 e 1980, como documentam várias publicações sobre a evolução dos e-journals no mundo (LANCASTER, 1995; HARTER; KIM, 1996; PEEK; POMERANTZ, 1998; BISHOP et al., 2000; KLING; CALLAHAN, 2002). No Brasil, os primeiros periódicos eletrônicos surgiram na década de 1990.

Entretanto, paralelamente ao surgimento e à evolução dos periódicos eletrônicos, prenunciava-se uma crise no modelo de editoração 
científica vigente, a qual viria a tornar insustentável a compra e o licenciamento de periódicos científicos por bibliotecas (ROWLANDS, 2007). Conforme ressaltam Amorim e Vergueiro (2006, p. 37), fatores econômicos "impulsionaram os grandes agentes distribuidores a contribuir para que o mercado de publicações periódicas impressas entrasse em uma crise sem precedentes no mundo...". Esses autores arrolam, com base nos trabalhos de Odlyzko (1997), Brakel (1995), Schauder (1994), Meyer (1997) e Rogers e Hurt (1990), algumas das causas deste fenômeno (CENDÓN et al., 2009):

a) Ineficácia do modelo tradicional: as informações científicas chegam ao seu público-alvo através de outros meios antes de sua efetiva publicação [...] (BRAKEL, 1995, p. 390);

b) Limites físicos: alguns artigos, que poderiam trazer informações novas e relevantes, acabam não sendo publicados, devido à falta de espaço nas edições impressas [...] (SCHAUDER, 1994, p.88);

c) Alta especialização e baixa circulação: há uma tendência global que torna excessivo o número atual de revistas científicas (algo típico da explosão informacional, característica das sociedades atuais), onde cada título, com raríssimas exceções, tem um público extremamente limitado e elitista [...] (BRAKEL, 1995, P. 390);

d) Altos e crescentes custos: a própria especialização e dificuldades ligadas à editoração forçam as publicações a terem tiragens pequenas $e$, como consequência, um baixo número de assinantes, criando, assim, um círculo vicioso que contribui para a elevação de custos e para a inflação dos preços observados, como indicam, em seus estudos, Brakel (1995), Schauder (1994), Meyer (1997) e, sobretudo, Odlyzko (1997);

e) Falta de espaço para armazenamento nas bibliotecas: o problema é comum às grandes universidades brasileiras, representando um custo adicional aos orçamentos locais (AMORIM; VERGUEIRO, 2006).

Nesse contexto, a forma eletrônica de publicação dos periódicos científicos foi vista como uma possível solução para os problemas que se apresentavam para editoras e para a comunidade científica, motivando iniciativas que objetivavam a viabilização do oferecimento das publicações em forma digital. Experiências, tais como o Projeto CORE (1990-1995), na Cornell University, com 20 periódicos da American Chemical Society; o Projeto TULIP (1991-1995), com 43 periódicos da Elsevier Science e Pergamon; o Projeto Red Sage (1992-1996), com 71 periódicos de 19 editoras; e o Projeto MUSE (1994-), com 300 periódicos de 60 editoras, 
exploraram o potencial do fornecimento de periódicos eletrônicos. Seus resultados foram utilizados pelas editoras de revistas científicas, tais como Academic Press, John Wiley, Elsevier Science, Springer-Verlag, Blackwell Scientific e grandes sociedades acadêmicas como o American Institute of Physics (BISHOP et al., 2000; PEEK, 1996), que, em torno de 1996, começaram a disponibilizar seus periódicos online, oferecendo aos assinantes acesso ao seu texto completo.

Nos dias atuais, a publicação de periódicos, em forma eletrônica, tem adquirido crescente volume. Já em 2001, dois terços das revistas científicas estavam disponíveis em ambos os formatos, impresso e eletrônico (TENOPIR, 2003). Sua aceitação e uso também têm sofrido progressivo aumento, conforme documentam as pesquisas realizadas. Periódicos que existem em ambos os formatos têm suas versões eletrônicas acessadas até oito vezes mais que as versões correspondentes impressas (ROWLANDS, 2007). As tendências parecem indicar a progressiva redução do uso do formato impresso (ROWLANDS, 2007).

\section{Portal de Periódicos da CAPES}

No Brasil, da mesma maneira que nos demais países do mundo nas décadas de 1980 e 1990, as coleções das bibliotecas universitárias vinham sendo afetadas pela crise do mercado editorial de publicações científicas. Os programas centralizados para aquisição de periódicos das instituições de ensino superior, iniciados em 1987, financiados pela CAPES e outros órgãos, tais como CNPq, Secretaria de Ensino Superior (SESU) e Financiadora de Estudos e Projetos (FINEP), passaram por interrupções e redução de recursos, levando, nestas instituições, a coleções incompletas, à diminuição no número de assinaturas de periódicos e ao impedimento de novas assinaturas.

A criação do consórcio de periódicos eletrônicos da CAPES, em 1999, visava solucionar esses problemas, através da disponibilização centralizada dos periódicos científicos para as instituições participantes, buscando a racionalização de recursos, a redução de custos por usuário e a otimização dos investimentos no acesso à produção científica mundial. A iniciativa, até aquele momento, dificultada por variadas limitações financeiras, foi viabilizada pela entrada massiva dos periódicos científicos eletrônicos no mercado brasileiro, em meados do ano 2000 (AMORIM; VERGUEIRO, 2006), culminando com a criação do Portal de Periódicos da Capes.

Desde o seu lançamento, o Portal tem sido objeto de pesquisas na área acadêmica. Cendón e Ribeiro (2008) traçaram, através do levantamento e análise de 40 trabalhos, um panorama destes estudos, onde se verificou que as pesquisas realizadas sobre o Portal podem ser classificadas, principalmente, em três grandes grupos: (a) estudos de uso e satisfação (por exemplo: MARTINS, 2006; MARTINS, FREIRE; PINHEIRO, 2006), (b) estudos do impacto do Portal na pesquisa e produtividade e de sua adequação (por exemplo: MUGNANI, 2006; FERNANDES; CENDÓN, 2010) e (c) estudos de usabilidade (por exemplo: 
ODDONE; MEIRELLES, 2006). Dentro desta classificação, a corrente pesquisa insere-se como uma contribuição para os estudos de uso e satisfação dos usuários do Portal CAPES.

\section{Medidas de sucesso de sistemas de informação}

A questão do sucesso de sistemas de informação e a satisfação dos seus usuários é apresentada, principalmente, em estudos de literatura estrangeira. Destes, citam-se, em particular, Ives, Olson e Baroudi. (1983), DeLone e McLean (1992), Rey Martin (2000) e Melone (1990), autores que fizeram levantamentos da literatura científica sobre o tema e sobre os fatores que levam à satisfação dos usuários e ao sucesso dos sistemas de informação.

Na busca de uma definição do sucesso de sistemas de informação, DeLone e McLean, em seu artigo de 1992, analisaram 100 trabalhos empíricos sobre o assunto e chegaram a um modelo síntese dessas pesquisas. Os autores se basearam na teoria de Shannon e Weaver (1949) e no trabalho de Mason (1978), para definir as diferentes formas usadas para medir o sucesso de sistemas de informação. Identificaram, classificaram e analisaram as medidas e definições de sucesso utilizadas em trabalhos publicados em sete periódicos da área de administração de empresa (Management Science, MIS Quarterly, Communications of the ACM, Decision Sciences, Information e Management, Journal of Management Information Systems e International Conference on Information Systems (ICIS) Proceedings), entre 1981 e 1988. Dessa análise, resultaram seis construtos interdependentes, que foram utilizados para propor um modelo de sucesso de sistemas de informação (FIG. 1):

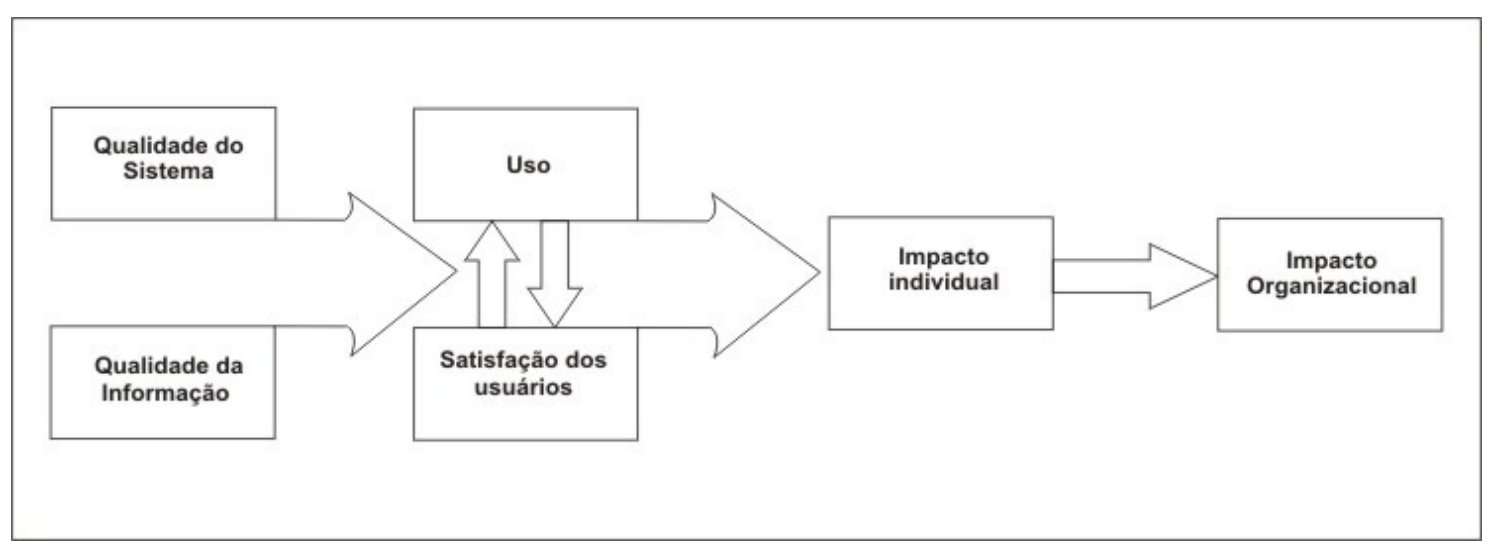

FIGURA 1 - Modelo de Sistema de Informação de Sucesso DeLone e McLean

Fonte: DELONE; MCLEAN, 1992.

No modelo de DeLone e Mclean (1992), os construtos qualidade do sistema e qualidade da informação, separada ou conjuntamente, afetam o construto uso e o construto satisfação do usuário. Além disso, a utilização frequente do sistema pelos usuários pode afetar o grau de satisfação do 
usuário de informação - positivamente ou negativamente. O construto uso e o construto satisfação do usuário são antecedentes diretos do construto impacto individual e, finalmente, o seu impacto sobre o desempenho individual deve, eventualmente, ter algum impacto organizacional, resultando no modelo apresentado na FIG. 1.

De acordo com DeLone e McLean (1992), a satisfação do usuário é, provavelmente, uma das mais importantes variáveis dependentes utilizadas para medir o sucesso do sistema de informação, devido a três razões. Primeiro, por ter a medida de satisfação um elevado grau de validade. Segundo, pelo desenvolvimento do instrumento de Bailey e Pearson e outros derivados dele, que proporcionaram instrumentos confiáveis para medir a satisfação do usuário. $\mathrm{E}$, terceiro, por ser difícil negar o sucesso de um sistema do qual os usuários dizem gostar (DELONE; MCLEAN, 1992).

Segundo Ives, Olson e Baroudi (1983), a visão da satisfação do usuário como um indicador de sucesso de um sistema, provavelmente originou-se com Cyert e March, em 1963. O conceito de satisfação proposto por Cyert e March (1963) sugere que o fato de um sistema de informação atender às necessidades do seu utilizador irá reforçar a satisfação do usuário com esse sistema. Se o sistema não fornecer as informações necessárias, o usuário ficará insatisfeito e procurará outra fonte de informação ou sistema de informação. Para Ives, Olson e Baroudi. (1983), a satisfação dos usuários de sistemas de informação é um mecanismo para sua avaliação. É definida como o grau em que, segundo a percepção dos usuários, os sistemas de informação satisfazem as suas necessidades de informação.

Rey Martín (2000) destaca que o termo "satisfação do usuário" ganhou atenção considerável na literatura acadêmica da área de Biblioteconomia e Ciência da Informação na década de 1980. Segundo a autora, a "satisfação do usuário com relação à operação e aos atributos de um sistema de informação está diretamente relacionada ao uso do sistema" (Rey Martín, 2000, p. 141).

Melone (1990, p. 79) ressalta, também, que as duas medidas de sucesso mais frequentes na literatura são a satisfação do usuário e o uso do sistema. Entre elas, a satisfação do usuário tem recebido maior atenção dos pesquisadores e "serve como o constructo primário pelo qual os sistemas de informação são avaliados e analisados". Independentemente de qual critério é selecionado, ou seja, a satisfação ou a utilização, esse critério deve ser justificado a partir da sua relação com a eficácia do sistema (MELONE, 1990). O autor menciona, também, o papel central que o construto satisfação do usuário ganhou nas investigações comportamentais sobre sistemas de informação. Destaca que, dentre as escalas mais populares de medição do construto satisfação do usuário está a de Bailey e Pearson (1983), discutida adiante, por medir de maneira mais precisa a eficácia de um sistema de informação.

Em sua revisão de literatura, Melone (1990) mostra que a falta de concordância no delineamento conceitual do construto satisfação do usuário tem levado à existência de inúmeras definições, sendo a maior 
parte delas, sem fundamento teórico. Definições específicas para o construto, tais como "a crença dos usuários em que os sistemas de informação satisfazem as suas necessidades de informação" têm em comum, a noção de que o usuário fornecerá algum tipo de resposta avaliativa sobre o sistema quando o utilizarem $O$ construto é, ainda, associado a uma variedade de termos tais como "aceitação do sistema", "utilidade percebida", "valorização do sistema" e "atitudes e percepções" dos usuários", entre outros (MELONE, 1990).

O termo "satisfação" aparece associado a dois outros termos: qualidade do sistema e avaliação dos usuários (Rey Martín, 2000). A avaliação dos sistemas, através da reação dos usuários, é feita para se melhorar a qualidade do sistema e, assim, alcançar a satisfação dos usuários. A satisfação com o sistema é um resultado que se pretende atingir e o uso do sistema depende tanto do serviço prestado como dos valores e expectativas dos usuários, bem como de outros fatores como o tempo, o custo e o esforço que o uso do sistema representa para os usuários. Os diferentes níveis de satisfação do usuário são determinados a partir da relação entre o serviço recebido e o esforço feito pelo usuário, resultando isso em quatro níveis de satisfação (Rey Martín, 2002), como mostra o QUADRO 1.

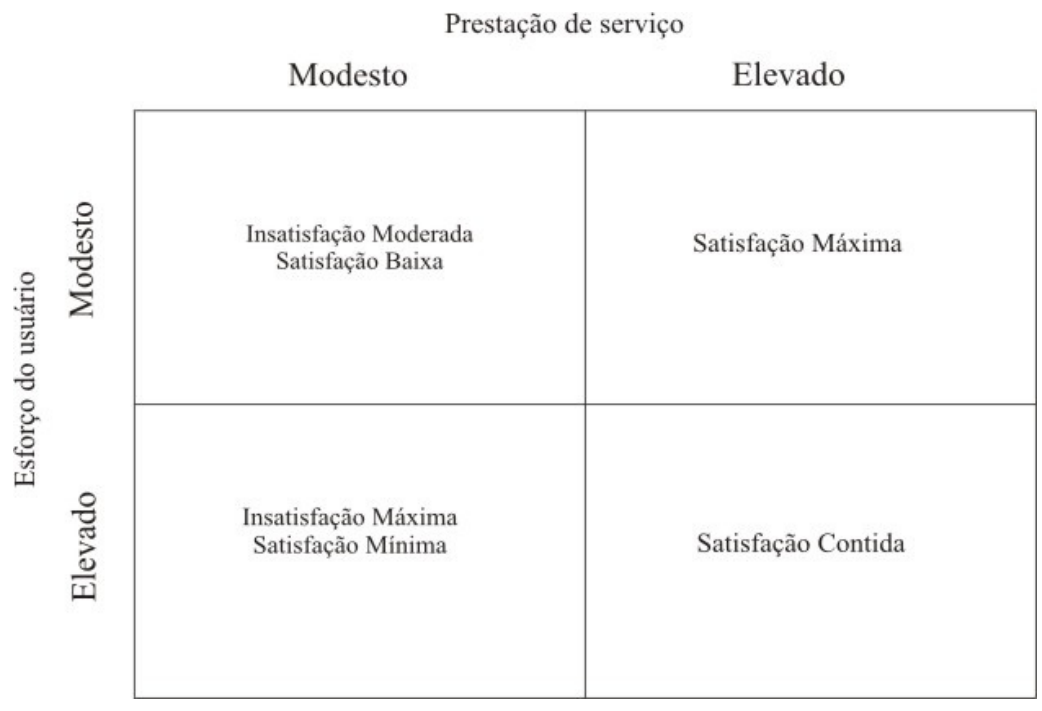

QUADRO 1 - Níveis de satisfação entre serviço e esforço

Fonte: Adaptado de REY MARTÍN, 2000.

As situações apresentadas, em cada setor do QUADRO 1, são:

1) Prestação de serviços modesta / Esforço modesto: esses fatores levam a uma satisfação ou insatisfação ligeiramente baixa, de modo que o julgamento do serviço pelo usuário traz incerteza sobre a sua utilização posterior do sistema;

2) Prestação de serviços modesta / Esforço elevado: a combinação destes dois fatores leva a um nível máximo de insatisfação ou a um nível mínimo de satisfação. O usuário faz uma apreciação negativa do serviço 
que pode dar origem a uma reclamação e a não utilização posterior do serviço;

3) Prestação de serviços elevada / Esforço elevado: esta combinação cria uma satisfação contida e um julgamento positivo pelo usuário. A incerteza sobre a posterior procura pelo serviço diminui, já que o usuário se dispõe a utilizar novamente o serviço; e

4) Prestação de serviços elevada / Esforço modesto: estes dois fatores levam a um nível máximo de satisfação. O julgamento é claramente positivo, o que permite uma maior confiança na posterior procura do serviço pelo usuário. Portanto, há um elemento que garante mais do que satisfação, a confiança do usuário. Se o sistema ganha a confiança do usuário, ele continua a utilizar o serviço e, muito provavelmente, o utilizará constantemente, se fidelizando.

A partir das quatro situações descritas, a autora conclui que a satisfação é um indicador com um forte componente subjetivo, porque é focado mais em percepções e atitudes do que em critérios concretos e objetivos. Rey Martín (2000, p. 143) destaca, ainda, que "do ponto de vista do usuário, a satisfação das suas necessidades de informação será a medida da eficácia do sistema, por excelência". As opiniões dos usuários sobre a satisfação ou insatisfação decorrem diretamente da qualidade do serviço que os sistemas oferecem, assim como de suas características e informações. De certa forma, a satisfação fornece uma avaliação da visão dos usuários sobre o sistema, e não uma avaliação da qualidade técnica desses sistemas, e pode levar a situações em que, se o usuário não encontra as informações que busca, um sistema de informação passa a ser percebido pelos usuários como ruim, insatisfatório ou deficiente. Dessa forma, é a satisfação da necessidade de informação do usuário que irá determinar o sucesso ou o fracasso de um sistema de informação.

Vários instrumentos foram desenvolvidos para medir a satisfação de usuários de sistemas de informação. Como já mencionado, um dos mais aceitos é o de Bailey e Pearson (1983), autores que utilizaram a literatura científica sobre o tema e a técnica do incidente crítico para identificar fatores, listados na íntegra no Anexo 2, relacionados à satisfação do usuário final de sistemas de computadores, tais como flexibilidade, integração do sistema, facilidade de uso, utilidade percebida, segurança dos dados, documentação, formato, relevância, precisão, idioma, pontualidade, velocidade, expectativas, entre outros. A satisfação do usuário com o sistema consiste na soma ponderada da sua reação positiva ou negativa em relação ao conjunto dos 39 fatores por eles determinados. Esse instrumento foi aplicado em uma organização com cinco unidades, para provar sua validade e confiabilidade. De acordo com Bailey e Pearson (1983, p. 538), a principal contribuição do seu trabalho "foi a definição de satisfação através de um instrumento de medição válido".

Ives, Olson e Baroudi. (1983) também apresentam uma contribuição em direção ao desenvolvimento de uma medida padrão de satisfação dos usuários de sistemas de informação. Após revisão de vários instrumentos para medida de satisfação, os autores consideraram o de Pearson (1977), como um potencial instrumento padrão para avaliação da satisfação dos 
usuários de sistemas de informação. Esta conclusão baseou-se na sua forte fundamentação empírica, no fato de poder ser o instrumento utilizado tanto para avaliação de produtos como de serviços de sistemas de informação e por permitir a determinação de múltiplos indicadores. Os autores fizeram validações adicionais do instrumento, através de um survey de uma amostra de gerentes de produção em organizações de manufatura dos Estados Unidos, e apresentaram sugestões para sua extensão e melhoria, no sentido de criar um padrão para medição da satisfação dos usuários de informações (IVES; OLSON; BAROUDI, 1983).

Para Rey Martin (2000) a satisfação do usuário pode ser tomada como um indicador da avaliação do desempenho e deve ser considerado como tal no planejamento do sistema de informação, considerando que a satisfação está diretamente relacionada com a utilização do sistema. Segundo a autora, pode-se perceber que o nível de utilização de um sistema de informações é uma indicação de satisfação do usuário para com o sistema, principalmente quando seu uso é opcional e não obrigatório, como no caso do Portal de Periódicos CAPES.

A seguir, é apresentada a metodologia do estudo, que utilizou principalmente uma abordagem quantitativa.

\section{Metodologia do estudo}

A metodologia utilizada no estudo foi principalmente quantitativa, com a coleta, também, de alguns dados qualitativos. Estes foram levantados através de um web-survey, enviado para docentes de todas as áreas do conhecimento distribuídos em 17 Universidades, nas cinco regiões brasileiras. Dos 5.176 docentes que se declararam usuários do Portal, analisaram-se as 930 respostas de docentes ao questionário sobre a satisfação dos usuários do Portal nas áreas de Ciências Biológicas, Ciências Humanas e Linguística, Letras e Artes.

A presente pesquisa optou por utilizar as mesmas áreas do conhecimento selecionadas por Cunha (2009), em sua dissertação de mestrado intitulada Uso de bibliotecas digitais de periódicos: um estudo comparativo no Portal de Periódicos CAPES entre as áreas do conhecimento. As áreas selecionadas foram escolhidas por apresentarem, respectivamente, baixo uso (Linguística, Letras e Artes), uso moderado (Ciências Humanas) e alto uso do Portal de Periódicos CAPES (Ciências Biológicas) (CUNHA, 2009; MAIA, 2005). As subáreas de cada uma dessas áreas, estão mencionadas na TAB. 1.

TABELA 1 - Áreas e subáreas selecionadas

$\begin{array}{clll}\text { Grande Área } & \begin{array}{c}\text { Ciências Biológicas } \\ \text { (7 subáreas) }\end{array} & \begin{array}{c}\text { Ciências Humanas } \\ \text { (9 subáreas) }\end{array} & \begin{array}{c}\text { Linguística, } \\ \text { Letras e Artes. } \\ \text { (3 subáreas) }\end{array} \\ \text { Subáreas } & \begin{array}{l}\text { Ciências Biológicas } \\ \text { Genética }\end{array} & \begin{array}{l}\text { Filosofia } \\ \text { Sociologia }\end{array} & \begin{array}{l}\text { Letras } \\ \text { Artes }\end{array} \\ & & & \end{array}$

Perspectivas em Ciência da Informação, v.16, n.2, p.67-100, abr./jun. 2011 


\begin{tabular}{lll|l}
\hline Zoologia & Antropologia & Música \\
Botânica & Arqueologia & \\
Biofísica & História & \\
Bioquímica & Geografia & \\
Ecologia & Psicologia & \\
& Educação & \\
& Ciência Política & \\
\hline
\end{tabular}

Fonte: Tabela de Áreas de Conhecimento do CNPq.

Os 930 respondentes do questionário sobre satisfação dos usuários do Portal, pertencentes às áreas selecionadas, estão assim distribuídos: 44\% são das Ciências Biológicas (405 respondentes); 41\% das Ciências Humanas (384 respondentes); e 15\% da área de Linguística, Letras e Artes (141 respondentes), conforme mostra o GRÁF. 1.

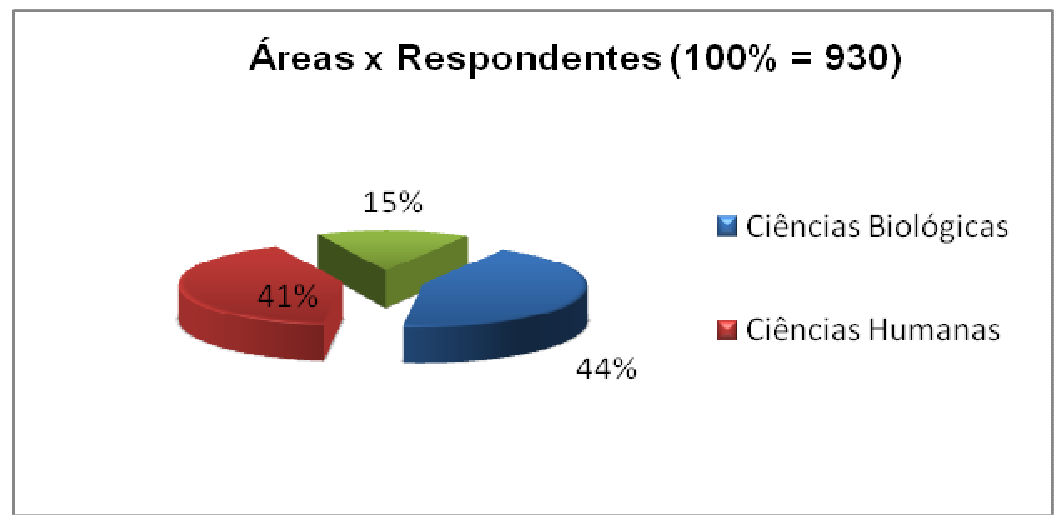

GRÁFICO 1 - Respondentes por Áreas do conhecimento

O questionário Pesquisa de Satisfação do Usuário do Portal CAPES continha 12 questões. O presente artigo analisa estatisticamente os dados coletados por duas destas, as quais foram escolhidas por serem relacionadas ao sucesso dos usuários na busca de informação no Portal e, consequentemente, à sua satisfação.

A primeira questão analisada visava verificar se o usuário obtinha sucesso ao procurar por um periódico específico, como mostra a FIG. 2:

\begin{tabular}{l|l}
\hline 9. Quando procura por um periódico específico no & OSim, freqüentemente $(75-100 \%$ das vezes) \\
Portal, você obtém sucesso? & OSim, quase sempre $(50-75 \%$ das vezes) \\
& OSim, às vezes ( $25-50 \%$ das vezes) \\
& OSim, raramente (menos de $25 \%$ das vezes) \\
& ONão obtenho( $0 \%)$ \\
& O O periódico não está disponivel \\
\hline
\end{tabular}

FIGURA 2 - Questão 9

A segunda questão analisada, também de múltipla escolha, tinha por objetivo verificar a razão do insucesso na busca por periódicos (FIG. 
3). Ela continha, também, um campo aberto para que o usuário descrevesse alguma outra resposta para a pergunta.

10. Se não obtém sucesso sempre quando procura por um periódico específico no Portal, qual a razão?

\section{OSempre obtenho sucesso}

O periódico não está disponivel

OA interface de busca é confusa

FIGURA 3 - Questão 10

Os dados quantitativos, obtidos nas respostas a estas questões, foram analisados através de estatística descritiva. Os dados qualitativos foram agrupados, conforme seu teor, em categorias.

\subsection{Apresentação dos resultados}

Dos 930 pesquisadores, 48\% (445) "quase sempre" (ou seja, entre $50 \%$ e $75 \%$ das vezes) obtêm sucesso na busca do periódico específico no Portal de Periódicos da CAPES e $22 \%$ (200), dizem que obtêm sucesso "frequentemente" (isto é, entre $100 \%$ e $50 \%$ das vezes), conforme mostra o Gráfico 2, a seguir. Dos respondentes, $23 \%$ obtêm sucesso em apenas $25 \%$ a $50 \%$ das vezes, ao recuperar um periódico específico no Portal e $5 \%$ raramente (isto é, menos de $25 \%$ das vezes) obtêm sucesso nas suas buscas. Em suma, $70 \%$ dos pesquisados obtêm um alto grau de sucesso no uso do Portal (entre $50 \%$ a $100 \%$ das vezes).

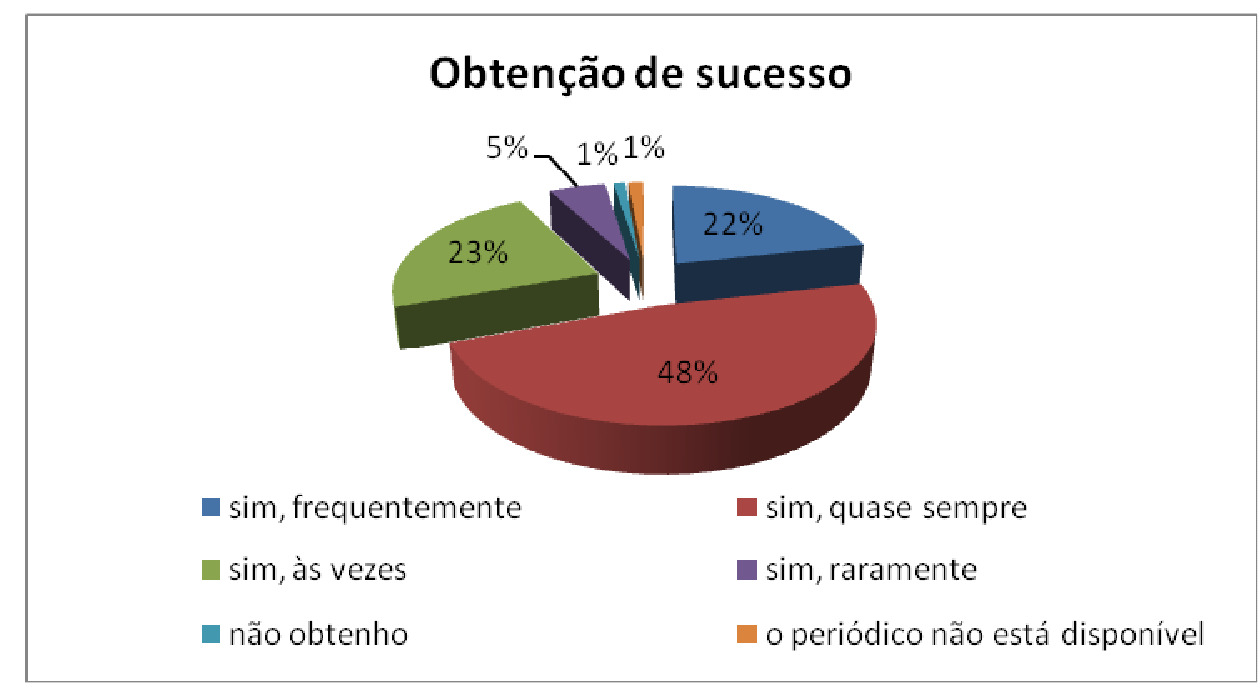

GRÁFICO 2 - Obtenção de sucesso

Conforme a TAB. 2, obtêm sucesso "quase sempre" $55 \%$ dos respondentes de Ciências Biológicas, $47 \%$ dos respondentes de Ciências Humanas e $30 \%$ dos respondentes de Linguística, Letras e Artes. Frequentemente obtêm sucesso na busca por periódicos (de $75 \%$ a $100 \%$ das vezes), $28 \%$ dos respondentes de Ciências Biológicas, $17 \%$ dos 
respondentes de Ciências Humanas e 20\% dos respondentes de Linguística, Letras e Artes. Nota-se, portanto, que $83 \%$ dos usuários de Ciências Biológicas e $63 \%$ dos usuários da área de Ciências Humanas obtêm sucesso de $50 \%$ a $100 \%$ das vezes. Para a área de Linguística, Letras e Artes, este índice de sucesso é obtido por menos da metade $(45 \%)$ dos usuários.

TABELA 2 - Áreas do conhecimento que obtêm sucesso quase sempre e frequentemente: análise por área

\begin{tabular}{|c|c|c|c|c|}
\hline Área & $\begin{array}{l}\text { Respondentes } \\
\text { por área }\end{array}$ & $\begin{array}{c}\text { Quase } \\
\text { sempre } \\
\text { N (\%) } \\
\text { (I) }\end{array}$ & $\begin{array}{c}\text { Frequentemente } \\
\text { N (\%) } \\
\text { (II) }\end{array}$ & $\begin{array}{c}\% \text { Total da área } \\
\mathbf{N}(\%) \\
(\mathrm{I}+\mathrm{II})\end{array}$ \\
\hline $\begin{array}{c}\text { Ciências } \\
\text { Biológicas }\end{array}$ & 405 & $223(55 \%)$ & $113(28 \%)$ & $336(83 \%)$ \\
\hline Ciências Humanas & 384 & 179 (47\%) & 67 (17\%) & 246 (64\%) \\
\hline $\begin{array}{l}\text { Linguística, } \\
\text { Letras e Artes }\end{array}$ & 141 & $43(30 \%)$ & $20(14 \%)$ & $63(45 \%)$ \\
\hline TOTAL & 930 & $445(48 \%)$ & $200(22 \%)$ & $645(70 \%)$ \\
\hline
\end{tabular}

Fonte: Dados da pesquisa.

A questão 10 - Se não obtém sucesso sempre quando procura por um periódico específico no Portal, qual a razão? - apresenta uma maioria de respondentes (76\%) que marcaram a opção "O periódico não está disponível", como razão pela qual não obtêm sucesso. Dos respondentes, $8,2 \%$ acreditam que "A interface de busca é confusa". Quase a mesma porcentagem dos respondentes ( $9 \%$ ) diz que há "outra razão" para não obtenção de sucesso. $\mathrm{E}, 7 \%$ dos respondentes, dizem que sempre obtém sucesso.

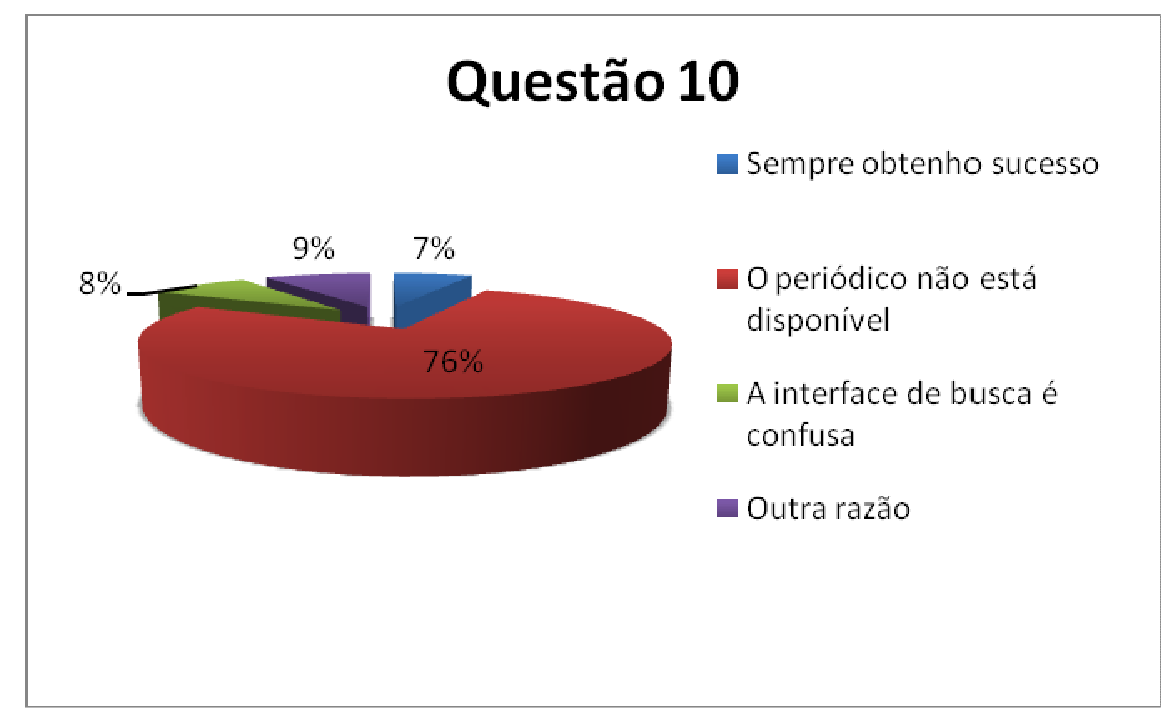

GRÁFICO 3 - Porcentagem das respostas da Questão 10 
Na TAB. 4, ao classificar os respondentes da Questão 10 por suas respectivas áreas do conhecimento, observamos que a área de Ciências Biológicas é a que apresenta o maior índice $(38,4 \%)$, em relação à resposta "o periódico não está disponível", seguida pela área de Ciências Humanas $(27,7 \%)$ e pela de Linguística, Letras e Artes $(10,0 \%)$.

TABELA 3 - Questão 10 (Distribuição dos respondentes por área do conhecimento)

\begin{tabular}{|c|c|c|c|c|c|}
\hline & $\begin{array}{l}\text { Sempre } \\
\text { obtenho } \\
\text { sucesso }\end{array}$ & $\begin{array}{c}\text { O } \\
\text { periódico } \\
\text { não está } \\
\text { disponível }\end{array}$ & $\begin{array}{c}\text { A interface } \\
\text { de busca é } \\
\text { confusa }\end{array}$ & Outra razão & Total \\
\hline Áreas & $\begin{array}{c}\text { Freq. } \\
\text { Absoluta } \\
(\%)\end{array}$ & $\begin{array}{c}\text { Freq. } \\
\text { Absoluta } \\
(\%)\end{array}$ & $\begin{array}{c}\text { Freq. } \\
\text { Absoluta } \\
(\%)\end{array}$ & $\begin{array}{c}\text { Freq. } \\
\text { Absoluta } \\
(\%)\end{array}$ & $\begin{array}{c}\text { Freq. } \\
\text { Absoluta } \\
(\%)\end{array}$ \\
\hline $\begin{array}{l}\text { Ciências } \\
\text { Biológicas }\end{array}$ & $19(2,0 \%)$ & $357(38,4 \%)$ & $7(0,8 \%)$ & $22(2,4 \%)$ & 405 (43,5\%) \\
\hline $\begin{array}{l}\text { Ciências } \\
\text { Humanas }\end{array}$ & $33(3,5 \%)$ & $257(27,7 \%)$ & $52(5,6 \%)$ & $42(4,5 \%)$ & $384(41,3 \%)$ \\
\hline $\begin{array}{l}\text { Linguística, } \\
\text { Letras e } \\
\text { Artes }\end{array}$ & $10(1,1 \%)$ & $94(10,0 \%)$ & $17(1,8 \%)$ & $20(2,2 \%)$ & $141(15,2 \%)$ \\
\hline Total & $62(6,7 \%)$ & 708 (76,1\%) & $76(8,2 \%)$ & $84(9,0 \%)$ & $\begin{array}{c}930 \\
(100,0 \%)\end{array}$ \\
\hline
\end{tabular}

Fonte: Dados da pesquisa.

Os dados da TAB. 4 e os GRÁF. 4, 5 e 6, abaixo, permitem concluir que dizem que "o periódico não está disponível" 357 ou seja $88,1 \%$ dos 405 respondentes da área de Ciências Biológicas (GRÁF. 4); 257, ou seja, $66,9 \%$ dos 384 respondentes de Ciências Humanas (GRÁF. 5); e 94, ou seja 66,7\% dos 141 respondentes da área de Linguística, Letras e Artes (GRÁF. 6).

O GRÁF. 4, a seguir, mostra a distribuição dos respondentes da área de Ciências Biológicas por suas subáreas. Desses, predominam os respondentes da subárea também denominada Ciências Biológicas. Notase que, em todas as subáreas, a razão acentuadamente preponderante para o insucesso da busca é a ausência do periódico. 


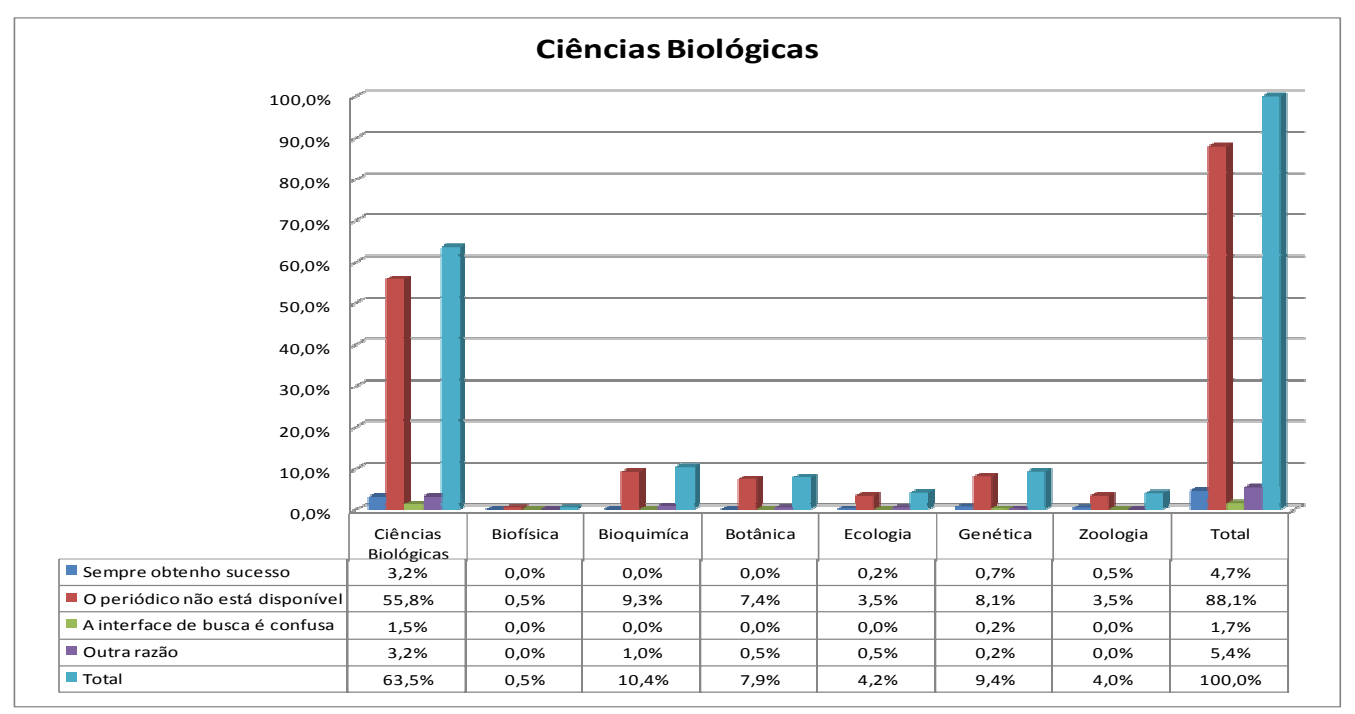

GRÁFICO 4 - Razão para não obtenção do periódico: respostas das subáreas de Ciências Biológicas

O mesmo ocorre na área de Ciências Humanas, em que respondentes das subáreas de Educação e de Psicologia predominam, mas prepondera, em todas as subáreas, como razão para o insucesso na busca, a ausência do periódico.

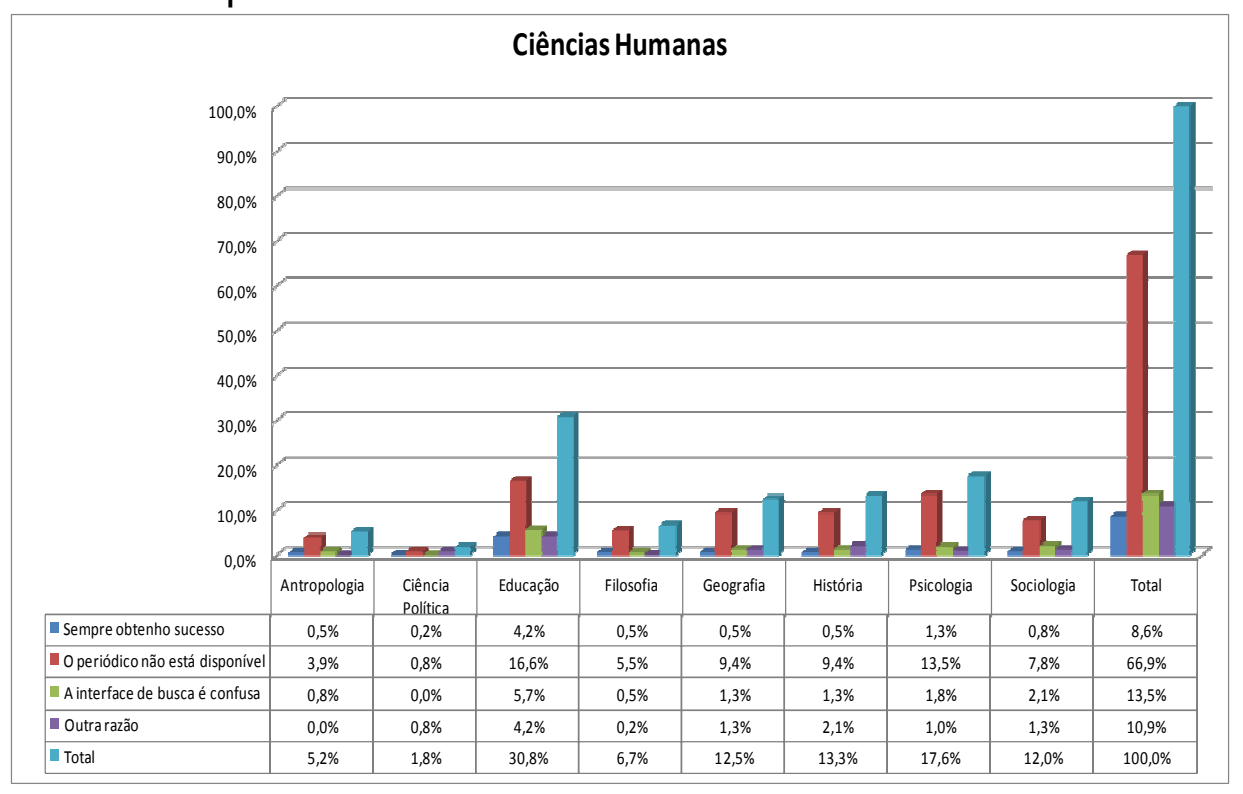

GRÁFICO 5 - Razão para não obtenção do periódico: respostas das subáreas da Ciências Humanas

$\mathrm{Na}$ área Linguística, Letras e Artes preponderam respondentes na subárea de Letras $(58,2 \%)$, mas, em todas as subáreas, o maior número de respostas indica ser a indisponibilidade do periódico $(66,7 \%$ das vezes, no total) a principal razão para o insucesso. 


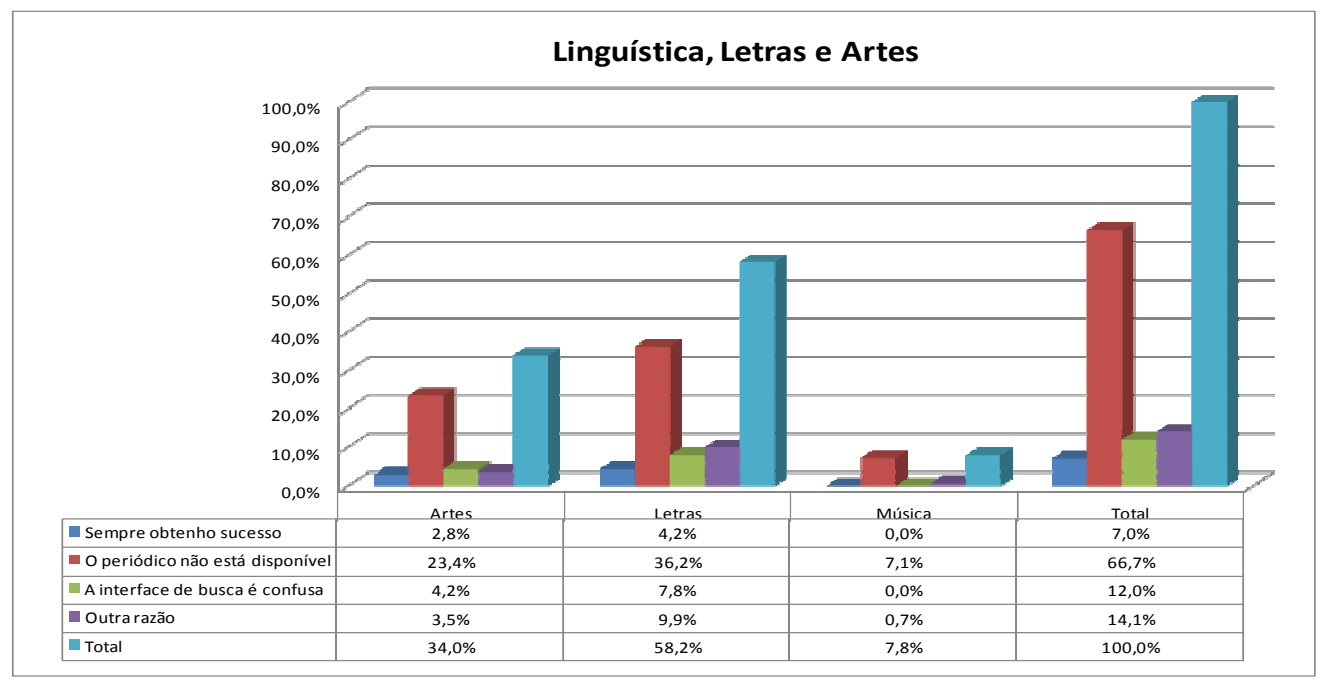

GRÁFICO 6 - Razão para não obtenção do periódico: Respostas das subáreas da Linguística, Letras e Artes

No campo "Outro", 56 pesquisadores $(6 \%$ do total de 930 respondentes) descreveram outros motivos para a não obtenção de sucesso, quando procuram por um periódico específico no Portal. Como podem ser visualizadas na Tabela 4, a seguir, essas razões foram classificadas em categorias.

TABELA 4 - Categorias das respostas da questão 10 do campo Outro

\begin{tabular}{llc}
\hline 1 & $\begin{array}{l}\text { Problemas com cobertura da base de dados de texto completo } \\
\text { (falta do periódico, falta de disponibilidade do texto completo, } \\
\text { falta de cobertura em determinadas áreas). }\end{array}$ & 33 \\
\hline 2 & $\begin{array}{l}\text { Dificuldades com os idiomas dos artigos. } \\
3\end{array}$ & $\begin{array}{l}\text { Limitações e dificuldades para acesso ao Portal (falta de senha, } \\
\text { Internet com problema, falta de permissão para acesso). }\end{array}$ \\
\hline 4 & $\begin{array}{l}\text { Problemas com a cobertura cronológica da base de dados (falta } \\
\text { do fascículo procurado, falta de artigos mais antigos em texto } \\
\text { completo). }\end{array}$ & 9 \\
\hline
\end{tabular}

Fonte: Dados da pesquisa.

A seguir, as respostas para cada uma das categorias, da TAB. 4, são detalhadas:

17 dos 32 respondentes que dizem ter "Problemas com a cobertura da base de dados de texto completo" dos periódicos no Portal de Periódicos CAPES alegam, por exemplo, como razão que "falta periódico da área" disponível no Portal ou que os periódicos "não existem", "não estão na base de dados", "o periódico não é assinado", "não está 
disponível", "o acervo é pobre", "falta do periódico", "às vezes o título não é incluído no Portal". Sobre a questão da não obtenção do texto completo, respondentes afirmam que "só conseguem o resumo e, na maioria das vezes, o texto completo não está disponível". Um pesquisador mencionou que "algumas revistas cobram o reprint". Dois pesquisadores da área de Linguística, Letras e Artes dizem que "algumas revistas importantes na área não estão disponíveis" e "periódicos da área de Letras, Linguística são poucos". Na área de Ciências Biológicas, pesquisadores reclamam que "não há muitas opções para quem trabalha com educação ambiental" e há "pouca coisa sobre meio ambiente". Na área de Ciências Humanas, um pesquisador escreveu que "o Portal é muito incompleto na área de Estudos Clássicos";

Com relação às "Dificuldades com os idiomas dos artigos", apenas um pesquisador diz não dominar a língua estrangeira e que isso dificulta a procura de periódicos sobre assuntos da área; Um pesquisador reclama da "absoluta ausência de periódicos relevantes em línguas francesa, italiana e espanhola";

Em relação às "Limitações e dificuldades para acesso ao Portal", os pesquisadores mencionaram como razões pelas quais não obtêm sucesso ao procurarem um periódico, que "o acesso ao Portal é limitado", "não é permitido o acesso / não tem acesso" e "não tenho senha para utilizar o Portal". Um pesquisador comentou que "o Portal estava fora do ar", o que ocorre devido a problemas com a Internet. Outro pesquisador disse que "desiste, mesmo que temporariamente, por falta de dispositivo alternativo". Alguns pesquisadores fazem referência a dificuldades pessoais, que podem ser identificadas a partir das suas respostas: "me atrapalho na busca", "às vezes tenho dúvida se busquei todas as possibilidades", "erro ao pesquisar no Portal" e "manuseio ineficiente". Há dois pesquisadores que mencionam problemas de usabilidade: "a interface de busca é rígida, não permite abreviações"; e

As respostas na categoria "Problemas com a cobertura cronológica das bases de dados" indicam comentários como "tempo de cobertura da base insuficiente", "estão disponíveis apenas os volumes mais recentes", "necessito de números mais antigos" e "há pouca atualização nos periódicos disponíveis". Pesquisadores identificam ainda que "há delay entre a disponibilidade da versão impressa e a eletrônica".

\section{Conclusão}

Este trabalho buscou medir a satisfação dos usuários do Portal de Periódicos da CAPES, a partir do seu sucesso na obtenção dos periódicos desejados. Através das respostas dos pesquisadores, identificam-se as dificuldades por eles experimentadas e nota-se que o nível de sucesso do usuário na busca de informação, no Portal de Periódicos da CAPES, varia conforme a área e subárea do conhecimento. Enquanto $83 \%$ e $64 \%$, respectivamente, dos usuários das áreas de Ciências Biológicas e Ciências Humanas obtêm sucesso na busca de periódicos frequentemente ou quase sempre (ou seja, entre $50 \%$ e $100 \%$ das suas buscas são bem sucedidas), 
menos da metade (45\%) dos usuários de Linguística, Letras e Artes, encontram, na maioria de suas pesquisas, o periódico que desejam.

Os dados encontrados apenas parcialmente corroboram os de Cunha (2009) que, em suas análises, concluiu que as dificuldades no uso da interface, a predominância de material em inglês no Portal e a ausência de materiais que se buscam no Portal são razões para a não utilização do Portal. No corrente estudo, ocorreu uma predominância expressiva de respostas ( $76 \%$ do total de respondentes) relacionadas à ausência do periódico no Portal.

As três áreas, Ciências Biológicas, Ciências Humanas e Linguística, Letras e Artes, indicaram, maciçamente, como razão principal para não obtenção de sucesso, a indisponibilidade do periódico ou de um determinado fascículo, no Portal: $88,1 \%$, 66,9\% e $66,7 \%$ dos respondentes de cada área, respectivamente, indicam essa como a razão para o insucesso na busca. O exame detalhado das subáreas mostra ser a ausência do texto buscado a razão preponderante para os casos de insucesso, em todas as subáreas.

A análise dos dados qualitativos, em que 56 docentes detalharam as razões para insucesso, confirmam esta constatação. Três, das quatro categorias em que as respostas foram organizadas, podem ser relacionadas à ausência do texto procurado no Portal, já que nas categorias 1, 2 e 4 os pesquisadores reclamam, por exemplo, da ausência de periódicos em outro idioma que não o inglês, da ausência de fascículos mais antigos, da presença de resumos apenas, da inexistência do texto completo, da falta de melhor cobertura em certas subáreas do conhecimento ou da ausência de periódicos mais importantes.

Apenas 8,2\% dos 930 respondentes assinalaram a opção "A interface de busca é confusa" como motivo para o insucesso, e $9 \%$ indicaram a opção "Outra razão". Observa-se (Gráficos 5 e 6) que houve incidência maior que a média da opção "A interface de busca é confusa", entre usuários de Ciências Humanas $(13,5 \%)$ e de Linguística, Letras e Artes $(12,1 \%)$, indicando a maior necessidade de treinamentos para esses usuários.

Os dados qualitativos não indicam que as dificuldades com os idiomas dos artigos sejam um problema relevante, já que apenas um pesquisador diz que a razão para insucesso relaciona-se à sua falta de domínio de língua estrangeira. Há, ainda, um número pequeno de pesquisadores (13 das 56 respostas qualitativas), que relatam dificuldades relacionadas às limitações para acesso ao Portal. Alguns dos comentários sugerem que essas dificuldades são devidas à necessidade de informação sobre o acesso ao Portal, já que alguns mencionam falta de autorização para acesso ou falta de senha, sendo que o acesso ao Portal é irrestrito para usuários das instituições pesquisadas. Essas respostas podem, também, ser indicativas de limitações de infraestrutura das instituições pesquisadas. Algumas poucas respostas, que mencionam dificuldades para lidar com a interface e para executar a busca, sugerem a necessidade de treinamento. 
A satisfação dos usuários é um mecanismo de avaliação de sistemas de informação e um indicador da crença pelos usuários do Portal de que este esteja cumprindo seu papel de atendimento das suas necessidades de informação. Os dados desta pesquisa indicam, como principal falha no atendimento às necessidades dos pesquisadores, a indisponibilidade de periódicos, o que, por sua vez, indica a necessidade de levantamentos sobre os periódicos necessários para cada área e subárea do conhecimento, e, ainda, de maiores investimentos na assinatura desses ou em assinaturas que permitam o acesso ao texto completo de fascículos mais antigos. Particular atenção merece a área de Linguística, Letras e Artes, em que menos da metade dos pesquisadores obtêm sucesso na busca em mais da metade das vezes em que procuram um periódico. Os dados também indicam que a falta de domínio de outros idiomas e, mesmo, a necessidade de treinamento, não representam maiores problemas para $76 \%$ dos respondentes.

\section{Referências}

AMORIM, A. M.; VERGUEIRO, W. Consórcios de bibliotecas no Brasil: um desafio à democratização do conhecimento. Perspectivas em Ciência da Informação, Belo Horizonte, v. 11, n. 1, p. 32-47, jan./abr. 2006.

BAILEY, J. E.; PEARSON, S. W. Development of a Tool for Measuring and Analyzing Computer User Satisfaction. Management Science, v. 29, n. 5, p. 530-545, May 1983.

BISHOP, A. P. et al. Digital libraries: situating use in changing information infrastructure. Journal of the American Society for Information Science, v. 51, n. 4, p. 394-413, 2000.

COORDENAÇÃO DE APERFEIÇOAMENTO DE PESSOAL DE NÍVEL SUPERIOR (CAPES). Portal. Disponível em: <www.periodicos.capes.gov.br $>$. Acesso em: 10 maio 2010.

Tabela de áreas de conhecimento. Disponível em: <http://www.capes.gov.br/avaliacao/tabela-de-areas-de-conhecimento $>$. Acesso em: 10 maio 2010.

BRAKEL, P. A. V. Electronic journal: publishing via Internet's WWW. Electronic Library, Bradford, v. 13, n.4, p. 389-395, 1995.

CENDÓN, B. V.; RIBEIRO, N. A. Análise da literatura acadêmica sobre o Portal Periódicos Capes. Informação \& Sociedade, João Pessoa, v. 18, p. 157-178, 2008. 
CENDÓN, B. V. et al. Uso dos periódicos do Portal CAPES pelos programas de pós-graduação da Faculdade de Odontologia de Araraquara - UNESP, no período de 2000 a 2005. Transinformação, Campinas, v. 21, n. 2, p.133-149, maio/ago. 2009.

CUNHA, A. Á. L. Uso de bibliotecas digitais de periódicos: um estudo comparativo no Portal de Periódicos CAPES entre as áreas do conhecimento. 2009. 207 f. Dissertação (Mestrado em Ciência da informação) - Escola de Ciência da informação, Universidade Federal de Minas Gerais, Belo Horizonte, 2009.

CYERT, R. M.; MARCH, J. G. A behavioral theory of the firm. Englewood Cliff, N. J.: Prentice-Hall, 1963.

DELONE, W. H.; MCLEAN, E. R. Information systems success: the quest for the dependent variable. Information Systems Research, Washington, v. 3, n. 1, p. 60-95, Mar. 1992.

FERNANDES, W. R.; CENDÓN, B. Ciência da Informação no Portal de Periódicos Capes: estudo bibliométrico para avaliação da coleção. In: SEMINARIO NACIONAL DE BIBLIOTECAS UNIVERSITARIAS (SNBU), 15., 2010, Rio de Janeiro = SEMINARIO INTERNACIONAL DE BIBLIOTECAS DIGITAIS, 2., 17-22 out. 2010, Rio de Janeiro. Anais.... Rio de Janeiro: UFRJ/SIBI; CRUESP, 2010.

HARTER, S. P.; KIM, H. J. Accessing electronic journals and other epublications: an empirical study. College and Research Libraries, v. 57, $\mathrm{n}$. 5 p. 440-456, Sep. 1996.

IVES, B.; OLSON, M. H. ; BAROUDI, J. J. The measurement of user information satisfaction. Communications of the ACM, New York, v. 26, n. 10, p. 785-793, Oct 1983.

KING, D. W.; TENOPIR, C. Using and reading scholarly literature. Annual Review of Information Science and Technology, New York, v. 34, p. 423$477,1999$.

KLING, R.; CALLAHAN, E. Electronic journals, the Internet and scholarly communication. Review of Information Science and Technology, v. 37, p. 127-177, 2003.

LANCASTER, F. W. The evolution of electronic publishing. Library Trends, v. 43, n. 4, p. 518-527, 1995.

MAIA, L. C. G. Uso de periódicos eletrônicos: um estudo sobre o Portal de Periódicos Capes na Universidade Federal de Minas Gerais. 2005. 153f. Dissertação (Mestrado em Ciência da Informação) - Escola de Ciência da Informação, Universidade Federal de Minas Gerais, Belo Horizonte, 2005.

MARTINS, M. F. M. Estudo do uso do Portal da Capes no processo de geração de conhecimento por pesquisadores da área biomédica: aplicando a técnica do incidente crítico. Rio de Janeiro, 2006. 126 f. Dissertação (Mestrado em Ciência da Informação) - Universidade Federal Fluminense / IBICT, Rio de Janeiro, 2006. 
MARTINS, M. F. M.; FREIRE, I. M.; PINHEIRO, L. V. R. O uso do Portal Capes na área biomédica: Uma abordagem qualitativa. In: CONFERENCIA IBEROAMERICANA DE PUBLICACOES ELETRONICAS NO CONTEXTO DA COMUNICACAO CIENTIFICA (CEPIC), 1., 2006, Brasília. Anais... Brasília: Programa de Pós-Graduação em Ciência da Informação da UnB, UNESP e UFF/IBICT, 2006. p. 165-172.

MASON, R. O. Measuring information output: A communication systems approach. Information \& Management, v. 1, n. 5, p. 219-234, Oct. 1978.

MEADOWS, A. J. A comunicação científica. Brasília: Briquet de Lemos, 1999.

MELONE, N. P. Theoretical Assessment of the User-Satisfaction Construct in Information Systems Research. Management Science, v. 36 n. 1, p. 76-91, Jan 1990.

MEYER, R. W. Monopoly power and electronic journals. Library Quarterly, Chicago, n. 67, n. 4, p. 325-349, 1997.

MILLER, S. Information-seeking behaviour of academic scientists in the electronic age. Ottawa: Canadian National Site License Project Evaluation Task Group, 2002. Disponível em: <http://www.cnslp.ca/initiatives/evaluation/LitReview-SusanMiller.pdf>. Acesso em: 12 ago. 2008.

ODDONE, N.; MEIRELLES, R. F. O Portal de Periódicos da CAPES e os indicadores de desempenho da informação eletrônica. Datagramazero, Rio de Janeiro, v. 7, n. 3, 2006. Disponível em: <www.dgz.org.br/jun06/Art 02.htm >. Acesso em: 12 ago. 2008.

ODLYZKO, A. The economics of eletronic journals. First Monday, Chicago, v. 2, n. 8, out. 2000. Disponível em: $<$ http:/firstmonday.org/issues/issue2 8/odlyzko/index.html $>$. Acesso em 10 jul. 2001.

PEARSON, S. Measurement of computer user satisfaction. (Ph.D. dissertation). Tempe: Arizona State University, 1977.

PEEK, R. Electronic publishing grows up. Journal of the American Society for Information Science, v. 47, n. 9, p. 665-668, 1996.

PEEK, R. P.; POMERANTZ, J. P. Electronic scholarly journal publishing. Annual Review of Information Science and Technology, v. 33, p. 321-356, 1998.

ROGERS, S.; HURT, C. S. How scholarly communication should work in the $21^{\text {st }}$ century. College and Research Libraries, v. 51, n.1, p. 5-8, 1990.

ROWLANDS, I. Electronic journals and user behavior: a review of recent research. Library \& Information Science Research, v. 29, p. 369-396, 2007.

SCHAUDER, D. Electronic publishing of professional articles: attitudes of academics and implications for the scholarly communication industry. 
Journal of the American Society for Information Science, New York, v. 45, n. 2, p. 73-100, 1994.

SHANNON, C. E.; WEAVER, W. A mathematical theory of communication. University of Illinois Press: Urbana Ill, 1949.

REY MARTIN, C. La satisfacción del usuário: un concepto en alza. Anales de Documentación, n. 3, p. 139-153, 2000.

TENOPIR, C. Use and users of electronic library resources: an overview and analysis of recent research studies. Washington, DC: Council on Library and Information Resources, 2003. Disponível em: <http://www.clir.org/pubs/abstract/pub120abst.html>. Acesso em: 4 set. 2009.

WANG, P. Methodologies and methods for user behavioural research. Annual Review of Information Science and Technology, v. 34, p. 53-59, 2001. 


\section{ANEXO 1 - Questionário Pesquisa de Satisfação dos Usuários do Portal CAPES}

\section{TERCEIRA ETAPA - PESQUISA DE SATISFAÇÃO}

\section{Respondente:}

Em nenhuma hipótese algum dado que permita identificar o respondente será divulgado.

Os dados pessoais serão utilizados apenas para evitar duplicidade.

\begin{tabular}{|l|l|}
\hline Nome: & \\
\hline E-mail: & \\
\hline Universidade & \\
\hline Escola / Instituto / Centro / Faculdade & \\
\hline Departamento & \\
\hline
\end{tabular}

\section{Satisfação em relação ao Portal de Periódicos CAPES}

\begin{tabular}{|c|c|c|c|c|c|c|c|c|c|c|c|}
\hline \multicolumn{6}{|c|}{$\begin{array}{l}\text { 1. Existe alguma dificuldade em sua utilização do Portal } \\
\text { de Periódicos CAPES? }\end{array}$} & \multicolumn{6}{|c|}{$\begin{array}{l}\text { OSim, muitas } \\
\text { OSim, poucas } \\
\text { ONão }\end{array}$} \\
\hline \multicolumn{12}{|c|}{ 2. Qual a sua reação geral ao Portal CAPES? } \\
\hline \multicolumn{12}{|c|}{ Sem opinião definida } \\
\hline \multirow[t]{2}{*}{0} & Péssimo & 0 & 0 & 0 & 0 & 0 & 0 & 0 & 0 & 0 & Excelente \\
\hline & & 0 & 1 & 2 & 3 & 4 & 5 & 6 & 7 & 8 & \\
\hline \multirow[t]{2}{*}{0} & Dificil & 0 & 0 & 0 & 0 & 0 & 0 & 0 & 0 & 0 & Fácil \\
\hline & & 0 & 1 & 2 & 3 & 4 & 5 & 6 & 7 & 8 & \\
\hline \multirow[t]{2}{*}{0} & Frustrante & 0 & 0 & 0 & 0 & 0 & 0 & 0 & 0 & 0 & Satisfatório \\
\hline & & 0 & 1 & 2 & 3 & 4 & 5 & 6 & 7 & 8 & \\
\hline \multirow[t]{2}{*}{0} & Rigido & 0 & 0 & 0 & 0 & 0 & 0 & 0 & 0 & 0 & Flexivel \\
\hline & & 0 & 1 & 2 & 3 & 4 & 5 & 6 & 7 & 8 & \\
\hline
\end{tabular}


Satisfação dos usuários do Portal de Periódicos da Capes: um estudo sobre a obtenção de sucesso no uso do sistema
Beatriz Valadares Cendón; Juliana Lopes de Almeida Souza; Nádia Ameno Ribeiro

Questionirio da Pesquiza Portal CAPES

3. Qual a sua reação ao design visual do Portal CAPES?

Sem opinião definida

O

De dificil leitura

O

0

\section{Caracteres na tela}

000000000

$\begin{array}{lllllllll}0 & 1 & 2 & 3 & 4 & 5 & 6 & 7 & 8\end{array}$

Organização da informação na tela

000000000

$\begin{array}{lllllllll}0 & 1 & 2 & 3 & 4 & 5 & 6 & 7 & 8\end{array}$

Sequiência de telas

000000000

$\begin{array}{lllllllll}0 & 1 & 2 & 3 & 4 & 5 & 6 & 7 & 8\end{array}$
De fácil leitura

Muito clara

Muito clara

\section{Qual a sua reação à divulgação e aprendizado do uso do Portal CAPES?}

Sem opinião definida

o

Inexistente

0

Insuficiente

O

0

○

$\circ$

Confuso

Nunca

\section{Treinamento no uso do Portal CAPES}

000000000

$\begin{array}{lllllllll}0 & 1 & 2 & 3 & 4 & 5 & 6 & 7 & 8\end{array}$

Divulgação do Portal CAPES

00000000000

Aprendizado para operar o sistema

000000000

$\begin{array}{lllllllll}0 & 1 & 2 & 3 & 4 & 5 & 6 & 7 & 8\end{array}$

Exploração do sistema por tentativa e erro

$$
000000000
$$

$\begin{array}{lllllllll}0 & 1 & 2 & 3 & 4 & 5 & 6 & 7 & 8\end{array}$

As tarefas podem ser realizadas de forma fácil e direta

000000000

$\begin{array}{lllllllll}0 & 1 & 2 & 3 & 4 & 5 & 6 & 7 & 8\end{array}$

Material de ajuda

000000000

$\begin{array}{lllllllll}0 & 1 & 2 & 3 & 4 & 5 & 6 & 7 & 8\end{array}$
Suficiente

Suficiente

Fácil

Fácil

Sempre

Claro

5. Qual a sua reação ao acesso ao Portal CAPES e seus periódicos?

Sem opinião definida

○

○

Muito lento

\section{Rapidez}

000000000

$\begin{array}{lllllllll}0 & 1 & 2 & 3 & 4 & 5 & 6 & 7 & 8\end{array}$

Impossivel

Suficientemente rápido

Imediato, conveniente e confiável
000000000

$\begin{array}{lllllllll}0 & 1 & 2 & 3 & 4 & 5 & 6 & 7 & 8\end{array}$ 
Satisfação dos usuários do Portal de Periódicos da Capes: um estudo sobre a obtenção de sucesso no uso do sistema
Beatriz Valadares Cendón; Juliana Lopes de Almeida Souza; Nádia Ameno Ribeiro

Questionirio da Pesquka Portal CApes

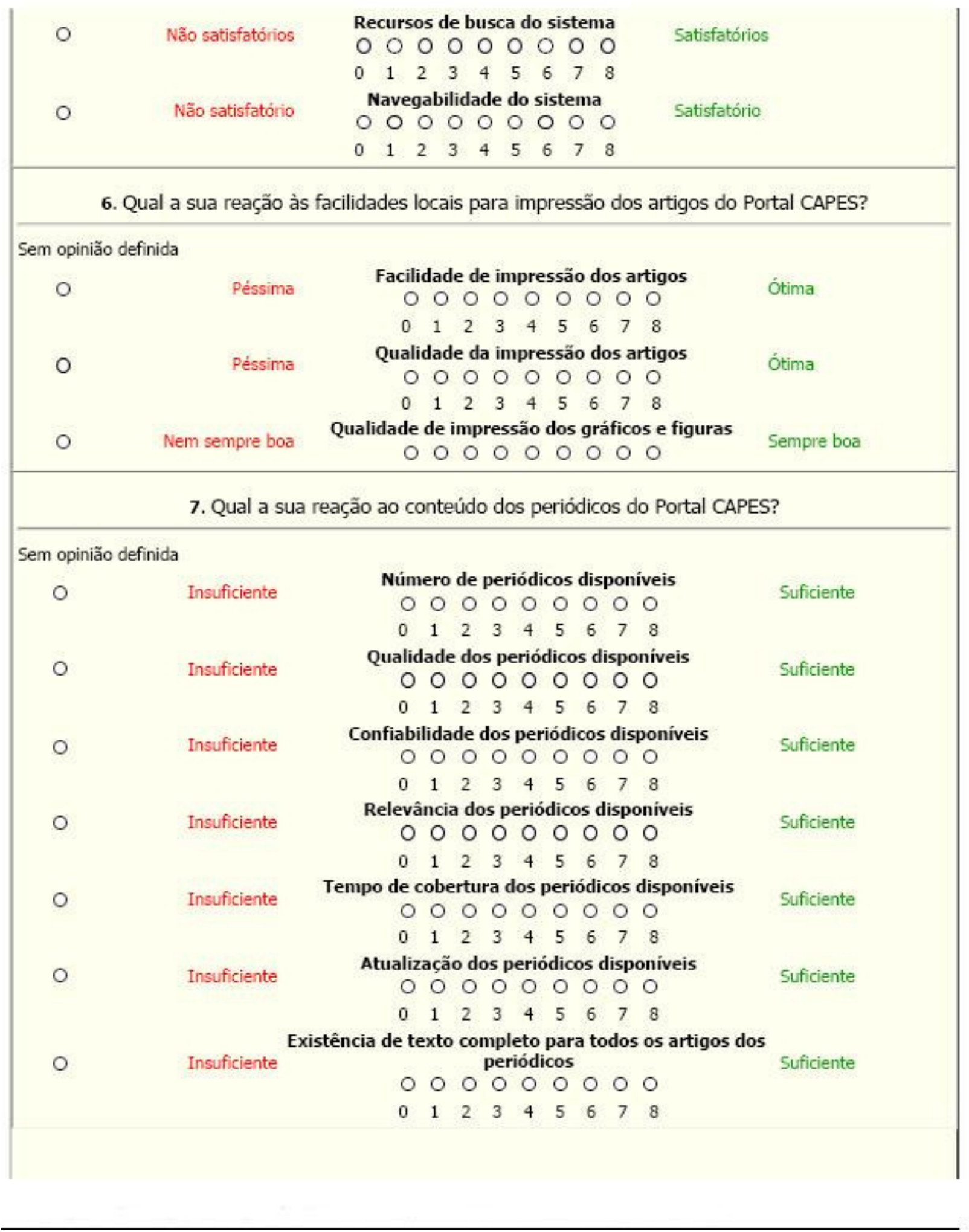


Satisfação dos usuários do Portal de Periódicos da Capes: um estudo sobre a obtenção de sucesso no uso do sistema
Beatriz Valadares Cendón; Juliana Lopes de Almeida Souza; Nádia Ameno Ribeiro

Questionirio da Resquisa Partal CAPES

\begin{tabular}{|c|c|}
\hline $\begin{array}{l}\text { 8. A existência de periódicos em idiomas os quais você } \\
\text { não domina prejudica a utilização do Portal CAPES. }\end{array}$ & $\begin{array}{l}\text { OConcordo totalmente } \\
\text { Oconcordo } \\
\text { Oconcordo moderadamente } \\
\text { O Indiferente } \\
\text { ODiscordo moderadamente } \\
\text { ODiscordo } \\
\text { ODiscordo totalmente }\end{array}$ \\
\hline $\begin{array}{l}\text { 9. Quando procura por um periódico específico no } \\
\text { Portal, você obtém sucesso? }\end{array}$ & $\begin{array}{l}\text { OSim, freqüentemente ( } 75-100 \% \text { das vezes) } \\
\text { OSim, quase sempre ( } 50-75 \% \text { das vezes) } \\
\text { OSim, às vezes ( } 25-50 \% \text { das vezes) } \\
\text { OSim, raramente (menos de } 25 \% \text { das vezes) } \\
\text { ONão obtenho( } 0 \% \text { ) } \\
\text { O periódico não está disponivel }\end{array}$ \\
\hline $\begin{array}{l}\text { 10. Se não obtém sucesso sempre quando procura por } \\
\text { um periódico especifico no Portal, qual a razão? }\end{array}$ & $\begin{array}{l}\text { OSempre obtenho sucesso } \\
\text { OO periódico não está disponivel } \\
\text { OA interface de busca é confusa } \\
\text { Outro: }\end{array}$ \\
\hline $\begin{array}{l}\text { 11. Em uma pesquisa simples de um assunto nas bases } \\
\text { de dados de resumos do Portal de Periódicos CAPES, } \\
\text { geralmente a informação e/ou material desejado é } \\
\text { encontrado? }\end{array}$ & $\begin{array}{l}\text { OSim, freqüentemente ( } 75-100 \% \text { das vezes) } \\
\text { OSim, quase sempre ( } 50-75 \% \text { das vezes) } \\
\text { OSim, às vezes ( } 25-50 \% \text { das vezes) } \\
\text { OSim, raramente (menos de } 25 \% \text { das vezes) } \\
\text { O Não obtenho( } 0 \% \text { ) } \\
\text { O Não utilizei }\end{array}$ \\
\hline $\begin{array}{l}\text { 12. Caso não tenha encontrado a informação nas } \\
\text { bases de dados, qual foi o motivo? }\end{array}$ & $\begin{array}{l}\text { OSempre encontro a informação } \\
\text { O Falta de familiaridade com a base de dados } \\
\text { O Dificuldade de usar as bases de dados } \\
\text { OA cobertura das bases de dados não é adequada } \\
\text { OA interface da editora da base de dados é confusa } \\
\text { ONão sei / não me lembro } \\
\text { Outro: }\end{array}$ \\
\hline $\begin{array}{l}\text { 13. Se possuir algum comentário ou dúvida sobre a } \\
\text { pesquisa acima, por favor utilize o espaço ao lado. }\end{array}$ & \\
\hline
\end{tabular}

Enviar dados 


\title{
ANEXO 2 - 39 fatores em relação à satisfação do usuário final $^{1}$
}

Top management involvement: The positive or negative degree of interest, enthusiasm, support, or participation of any management level above the user's own level toward computer-based information systems or services or toward the computer staff which supports them.

\author{
strong vs weak \\ consistent vs inconsistent \\ good vs bad \\ significant vs insignificant
}

Organizational competition with EDP unit: The contention between the respondent's organizational unit and the EDP unit when vying for organizational resources or for responsibility for success or failure of computer-based information systems or services of interest to both parties.
productive vs destructive
rational vs emotional
low vs high
harmonious vs dissonant

Priorities determination: Policies and procedures which establish precedence for the allocation of EDP resources and services between different organizational units and their requests.

fair vs unfair

consistent vs inconsistent

just vs unjust

precise vs vague

Charge-back method of payment for service: The schedule of charges and the procedures for assessing users on a pro rata basis for the EDP resources and services that they utilize.

just vs unjust

reasonable vs unreasonable

consistent vs inconsistent

known vs unknown

Relationship with the EDP staff: The manner and methods of interaction, conduct, and association between the user and the EDP staff.

\footnotetext{
${ }^{1}$ BAILEY; PEARSON, 1989, p. 539-543.
} 


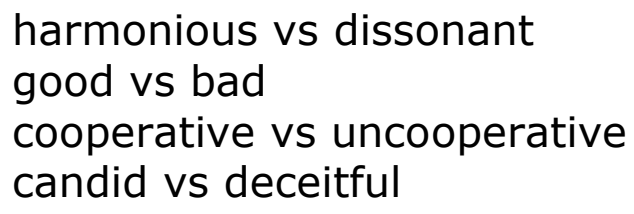

Comumunication with the EDP slaff: The manner and methods of information exchange between the user and the EDP staff .

harmonious vs dissonant

productive vs destructive

precise vs vague

meaningful vs meaningless

Technical competence of the EDP staff : The computer technology skills and expertise exhibited by the EDP staff.

current vs obsolete

sufficient vs insufficient

superior vs inferior

high vs low

Attitude of the EDP staff: The willingness and commitment of the EDP staff to subjugate external, professional goals in favor of organizationally directed goals and tasks.

user-oriented vs self-centered

cooperative vs belligerent

courteous vs discourteous

positive vs negative

Schedule of products and services: The EDP center timetable for production of information system outputs and for provision of computerbased services.

good vs bad

regular vs irregular

reasonable vs unreason able

acceptable vs unacceptable

Time required for new development: The elapsed time between the user's request for new applications and the design, development, and/or implementation of the application systems by the EDP staff.

short vs long

dependable vs undependable

reasonable vs unreasonable

acceptable vs unacceptable 
Processing of change requests: The manner, method, and required time with which the EDP staff responds to user requests for changes in existing computer-based information systems or services.

fast vs slow

timely vs untimely

simple vs complex

flexible vs rigid

Vendor support: The type and quality of the service rendered by a vendor, either directly or indirectly, to the user to maintain the hardware or software required by that organizational status.

skilled vs bungling

sufficient vs insufficient

eager vs indifferent

consistent vs inconsistent

Response/ turnaround time: The elapsed time between a userinitiated request for service or action and a reply to that request. Response time generally refers to the elapsed time for terminal type request or entry. Turnaround time generally refers to the elapsed time for execution of a program submitted or requested by a user and the return of the output to that user.

fast vs slow

good vs bad

consistent vs inconsistent

reasonable vs unreasonable

Means of input / output with EDP center: The method and medium by which a user inputs data to and receives output from the EDP center.

convenient vs inconvenient

clear vs hazy

efficient vs inefficient

organized vs disorganized

Convenience of access: the ease or difficulty with which the user may act to utilize the capability of the computer system.

convenient vs inconvenient

good vs bad

easy vs difficult

efficient vs inefficient

Accuracy: The correctness of the output information. 
accurate vs inaccurate

high vs low

consistent vs inconsistent

sufficient vs insufficient

Timeliness: The availability of the output information at a time suitable for its use.

timely vs untimely

reasonable vs unreasonable

consistent vs inconsistent

punctual vs tardy

Precision: The variability of the output information from that which it purports to measure.

sufficient vs insufficient

consistent vs inconsistent

high vs low

definite vs uncertain

Reliability: The consistency and dependability of the output information.

consistent vs inconsistent

high vs low

superior vs inferior

sufficient vs insufficient

Currency: The age of the output information.

good vs bad

timely vs untimely

adequate vs inadequate

reasonable vs unreasonable

Completeness: The comprehensiveness of the output information content.

complete vs incomplete consistent vs inconsistent

sufficient vs insufficient

adequate vs inadequate

Format of output: The material design of the layout and display of the output contents. 
good vs bad

simple vs complex

readable vs unreadable

useful vs useless

Language: The set of vocabulary, syntax, and grammatical rules used to interact with the computer systems.

simple vs complex

powerful vs weak

easy vs difficult

easy-to-use vs hard-to-use

Volume of output: The amount of information conveyed to a user from computer-based systems. This is expressed not only by the number of reports or outputs but also by the voluminousness of the output contents.

concise vs redundant

sufficient vs insufficient

necessary vs unnecessary

reasonable vs unreasonable

Relevancy: The degree of congruence between what the user wants or requires and what is provided by the information products and services.

useful vs useless

relevant vs irrelevant

clear vs hazy

good vs bad

Error recovery: The methods and policies governing correct ion and rerun of system outputs that all incorrect.

fast vs slow

superior vs inferior

complete vs incomplete

simple vs complex

Security of data: The safeguarding of data from misappropriation or unauthorized alteration or loss.

secure vs insecure

good vs bad

definite vs uncertain

complete vs incomplete 
Documentation: The recorded description of an information system. This includes formal instructions for the utilization of the system.
clear vs hazy
available vs unavailable
complete vs incomplete
current vs obsolete

Expectations: The set of attributes or features of the computerbased information products or services that a user considers reasonable and due from the computer-based information support rendered within his organization.

\author{
pleased vs displeased \\ high vs low \\ definite vs uncertain \\ optimistic vs pessimistic
}

Understanding of systems: The degree of comprehension that a user possesses about the computer-based information systems or services that are provided.
high vs low
sufficient vs insufficient
complete vs incomplete
easy vs hard

Perceived utility: The user's judgment about the relative balance between the cost and the considered usefulness of the computer-based information products or services that are provided. The costs include any costs related to providing the resource, including money, time, manpower, and opportunity. The usefulness includes any benefits that the user believes to be derived from the support.

high vs low

positive vs negative

sufficient vs insufficient

useful vs useless

Confidence in the systems: The user's feelings of assurance or certainty about the systems provided.
high vs low
strong vs weak
definite vs uncertain
good vs had 
Feeling of participation: The degree of involvement and commitment which the user shares with the EDP staff and others toward the functioning of the computer-based information systems and services.

positive vs negative

encouraged vs repelled

sufficient vs insufficient

involved vs uninvolved

Feeling of control: The user's awareness of the personal power to regulate, direct or dominate the development, alteration, and / or execution of the computer-based information systems or services which serve the user's perceived function.

high vs low

sufficient vs insufficient

precise vs vague

strong vs weak

Degree of training: The amount or specialized instruction and practice that is afforded to the user to increase the user's proficiency in utilizing the computer capability that is unavailable.

complete vs incomplete

sufficient vs insufficient

high vs low

superior vs inferior

Job effects: The changes in job freedom and job performance that are ascertained by the user as resulting from modifications induced by the computer-based information systems and services.

liberating vs inhibiting

significant vs insignificant

good vs bad

valuable vs worthless

Organization Position of the EDP Function: The hierarchical relationship of the EDP function to the overall organizational structure.

appropriate vs inappropriate

strong vs weak

clear vs hazy

progressive vs regressive

Flexibility of Systems: The capacity of the information system to change or to adjust in response to new conditions, demands, or circumstances. 
flexible vs rigid

versa tile vs limited

sufficient vs insufficient

high vs low

Integration of systems: The ability of systems to communicate / transmit data between systems servicing different functional areas.

complete vs incomplete

sufficient vs insufficient

successful vs unsuccessful

good vs bad 